\title{
分光光度法研究可逆反应动力学过程
}

黄希哲, 廖增俊, 何心怡, 许荣添, 韩东梅 ${ }^{*}$

中山大学化学工程与技术学院, 广东珠海 519082

摘要: 动力学实验是物理化学实验教学中的重要组成部分。利用分光光度法研究酚酞- $\mathrm{NaOH}$ 体系可逆动力学反应过 程, 实验结果表明环境温度对该实验反应趋势影响不大, 仅使用分光光度计一种仪器即可完成实验教学。该实验所 用试剂毒性低、用量少, 符合绿色化学实验理念。

关键词: 分光光度法; 可逆反应; 动力学; 氢氧化钠; 酚酞

中图分类号: G64; O6

\section{Spectrophotometric Method for Kinetics of Reversible Reaction}

Xizhe Huang, Zengjun Liao, Xinyi He, Rongtian Xu, Dongmei Han *

School of Chemical Engineering and Technology, Sun Yat-Sen University, Zhuhai 519082, Guangdong Province, China.

Abstract: Kinetics is an important part of physical chemistry laboratory teaching. In this article, we present a spectrophotometric method for study of the reversible kinetic reaction process of phenolphthalein- $\mathrm{NaOH}$ system. The ambient temperature has little influence on the experimental reaction, and the spectrophotometer is the only required instrument for carrying out this experiment. All reagents used are in small amount and low toxic, which is in accordance with the green chemistry concept.

Key Words: Spectrophotometric method; Reversible reaction; Dynamics; Sodium hydroxide; Phenolphthalein

可逆反应是指在同一条件下同时发生正逆反应的现象, 其反应过程会涉及化学平衡及化学反应 速率的问题, 是一类重要的反应类型, 但目前高校的物理化学实验课程中基本没有涉及可逆反应动 力学的相关实验教学 ${ }^{[1-4]}$ 。此外, 开展绿色化学实验教学是当今实验教学改革的重点之一 ${ }^{[5]}$, 本文 将介绍一个符合绿色化学实验教学理念的实验体系——分光光度法研究酚酞 $-\mathrm{NaOH}$ 体系可逆动力学 反应过程 $[6,7]$, 并研究酚酞浓度、温度对其动力学过程的影响。

\section{1 原理讨论}

酚酞在碱性溶液中由内酯式快速转化为醌式(快反应), 之后在醌式(A)与甲醇式(B)之间慢慢互 相转化(慢反应) ${ }^{[8]}$, 最终达到平衡状态(图1)。 


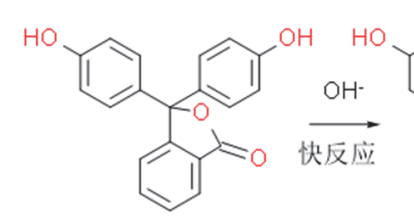

内酯式

无色

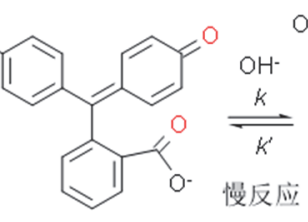

醌式

红色

(A)

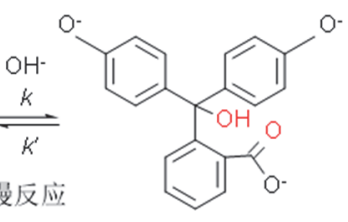

甲醇式

无色

(B)

图1 酚酞在弱碱性条件下的结构式转化情况

整个反应的动力学速率由醌式-甲醇式 $(\mathrm{A} \rightleftharpoons \mathrm{B})$ 转化速率决定, 设其正、逆反应速率常数分别为 $k$ 和 $k^{\prime}, \mathrm{A}$ 初始浓度为 $a, \mathrm{OH}^{-}$的初始浓度为 $b, T=t$ 时刻 $\mathrm{A} 、 \mathrm{~B}$ 浓度为 $x_{\mathrm{A} t} 、 x_{\mathrm{B} t}, T=\infty$ 时 $\mathrm{A} 、 \mathrm{~B}$ 浓度为 $x_{\mathrm{A} \infty}$ 、 $x_{\mathrm{B} \infty}$, 由Nicholson的工作 ${ }^{[7]}$ 可知 $b>>a$ 情况下, 正逆反应为一级反应, 故这里定义各种反应物的量级 为 1 , 可得:

$$
\begin{aligned}
& T=0 \\
& \mathrm{~A}^{-}+\mathrm{OH}^{-} \leftrightharpoons \mathrm{B}^{2-} \\
& T=t \\
& a \\
& T=\infty \\
& x_{\mathrm{A} t}=a-x_{\mathrm{B} t} \\
& b \\
& b-x_{\mathrm{B} t} \\
& x_{\mathrm{B} t} \\
& x_{\mathrm{A} \infty}=a-x_{\mathrm{B} \infty} \\
& x_{\mathrm{B} \infty}
\end{aligned}
$$

速率方程为: $\frac{\mathrm{d} x}{\mathrm{~d} t}=k\left(a-x_{\mathrm{B} t}\right)\left(b-x_{\mathrm{B} t}\right)-k^{\prime} x_{\mathrm{B} t}$

当 $a<<b$ 时, 可推出 $x_{\mathrm{B} t}<a<<b$, 可得: $\frac{\mathrm{d} x}{\mathrm{~d} t}=k b\left(a-x_{\mathrm{B} t}\right)-k^{\prime} x_{\mathrm{B} t}=k a b-\left(k b+k^{\prime}\right) x_{\mathrm{B} t}$

对其进行积分整理可得:

$$
x_{\mathrm{B} t}=\frac{k a b}{k b+k^{\prime}}\left\{1-\exp \left[-\left(k b+k^{\prime}\right) T\right]\right\} \Longrightarrow \exp \left[-\left(k b+k^{\prime}\right) T\right]=1-\frac{k b+k^{\prime}}{k a b} x_{\mathrm{B} t}
$$

当 $T=\infty$ 时, 由于 $x_{\mathrm{A} \infty}=a-x_{\mathrm{B} \infty}$, 可得:

$$
x_{\mathrm{B} \infty}=\frac{k a b}{k b+k^{\prime}} \Longrightarrow x_{\mathrm{A} \infty}=a-x_{\mathrm{B} \infty}=a-\frac{k a b}{k b+k^{\prime}}=\frac{k^{\prime} a}{k b+k^{\prime}}
$$

将(4)式代入(3)式且由于 $x_{\mathrm{A} t}=a-x_{\mathrm{B} t}, x_{\mathrm{A} \infty}=a-x_{\mathrm{B} \infty}$ 可得:

$$
\exp \left[-\left(k b+k^{\prime}\right) T\right]=1-\frac{x_{\mathrm{B} t}}{x_{\mathrm{B} \infty}}=\frac{x_{\mathrm{B} \infty}-x_{\mathrm{B} t}}{x_{\mathrm{B} \infty}}=\frac{a-x_{\mathrm{A} \infty}-\left(a-x_{\mathrm{A} t}\right)}{a-x_{A \infty}}=\frac{x_{\mathrm{A} t}-x_{\mathrm{A} \infty}}{a-x_{\mathrm{A} \infty}}
$$

根据朗伯比尔定律 $A=\varepsilon l c$ 可知在低浓度溶液中 $A \propto c$, 设 $A_{0}$ 为 $T=0$ 时对应的吸光度(此时 $\mathrm{A}$ 的 浓度为 $a), A_{t}$ 为 $x_{\mathrm{A} t}$ 对应的吸光度, $A_{\infty}$ 为 $x_{\mathrm{A} \infty}$ 对应的吸光度, 结合(5)式可得:

$$
\exp \left[-\left(k b+k^{\prime}\right) T\right]=\frac{A_{t}-A_{\infty}}{A_{0}-A_{\infty}} \Longrightarrow A_{t}=A_{\infty}+\left(A_{0}-A_{\infty}\right) \exp \left[-\left(k b+k^{\prime}\right) T\right]
$$

将(6)式求对数整理可得:

$$
\ln \left(\frac{A_{0}-A_{\infty}}{A_{t}-A_{\infty}}\right)=\left(k b+k^{\prime}\right) T
$$

$A \propto c$ 结合(4)式可得：

$$
x_{\mathrm{B} \infty}=a-x_{\mathrm{A} \infty}=a\left(1-\frac{x_{\mathrm{A} \infty}}{a}\right)=a\left(1-\frac{A_{\infty}}{A_{0}}\right)=\frac{k a b}{k b+k^{\prime}} \Longrightarrow \frac{A_{0}-A_{\infty}}{A_{0}}=\frac{k b}{k b+k^{\prime}}
$$

获得 $k$ 和 $k^{\prime}$ 有两种方法:

1 、将实验数据用Origin软件作Exponential拟合并结合(6)、(8)式可求得 $k$ 和 $k$ '。 
2、将实验数据做 $\ln \left(\frac{A_{0}-A_{\infty}}{A_{t}-A_{\infty}}\right)-T$ 图, 根据斜率并结合 $(8)$ 式可求得 $k$ 和 $k$ 。

\section{2 实验试剂及仪器}

\section{1 试剂}

$0.05 \mathrm{~mol} \cdot \mathrm{L}^{-1}$ 酚酞乙醇溶液、 $0.5 \mathrm{~mol} \cdot \mathrm{L}^{-1} \mathrm{NaOH}$ 溶液(经邻苯二甲酸氢钾标定)、去离子水。

\section{2 仪器}

美国Thermo Evolution 201紫外-可见分光光度计(带冷却水循环系统)、1 cm石英比色㿼。

\section{3 实验步骤}

\section{1 配制 $0.05 \mathrm{~mol} \cdot \mathrm{L}^{-1} \mathrm{NaOH}$ 溶液}

准备三支 $50 \mathrm{~mL}$ 比色管, 分别准确移入 $5 \mathrm{~mL} 0.5 \mathrm{~mol} \cdot \mathrm{L}^{-1} \mathrm{NaOH}$ 溶液并用去离子水定容到 $50 \mathrm{~mL}$ 。

\section{2 获取 430-620 $\mathrm{nm}$ 吸收曲线}

紫外分光光度计于 $25^{\circ} \mathrm{C}$ 恒温条件下通水半小时以上, 采用扫描测量模式, 测量波长设置为430$620 \mathrm{~nm}$, 用第一份 $\mathrm{NaOH}$ 溶液(空白)进行基线扫描。取第二份 $\mathrm{NaOH}$ 溶液准确加入 $0.1 \mathrm{~mL} 0.05 \mathrm{~mol} \cdot \mathrm{L}^{-1}$ 酚酞乙醇溶液(此时, $a=0.1 \mathrm{mmol} \cdot \mathrm{L}^{-1}, \quad b=0.05 \mathrm{~mol} \cdot \mathrm{L}^{-1}$ )迅速摇匀, 加入溶液的瞬间开始计时, 迅速 转入比色血并放入仪器, 当计时为 $5 \mathrm{~min}$ 时开始扫描获取吸收曲线数据(图2)。由图 2 可知反应体系的 最大吸收波长为 $553 \mathrm{~nm}$.

\section{3 获取动力学吸收曲线}

紫外分光光度计于 $25^{\circ} \mathrm{C}$ 恒温条件下通水半小时以上, 采用动力学分析模式, 测量波长为 $553 \mathrm{~nm}$ (由上一步骤获得), 采样时长为 $1 \mathrm{~h}$, 采用步长为 $30 \mathrm{~s}$ 。用第一份 $\mathrm{NaOH}$ 溶液(空白)进行较准归零。取 第三份 $\mathrm{NaOH}$ 溶液准确加入 $0.1 \mathrm{~mL} 0.05 \mathrm{~mol} \cdot \mathrm{L}^{-1}$ 酚酞乙醇溶液, 迅速摇匀 (此时溶液的吸光度为 $A_{0}$ ), 加入溶液的瞬间开始计时, 迅速转入比色典并放入仪器进行数据采集, 同时记下摇匀试剂到开始 采集数据时长(用于反应时间的修正)。将获得的数据用Origin软件绘图, 并进行Exponential拟合(图3), 可得拟合方程: $A_{t}=0.6252+2.479 \exp (-0.039 T)$, 由图3结合(6)式可得 $A_{\infty}=0.6252 、 A_{0}-A_{\infty}=2.479$ 、 $k b+k^{\prime}=0.039$ 。已知 $a=0.1 \mathrm{mmol} \cdot \mathrm{L}^{-1}, b=0.05 \mathrm{~mol} \cdot \mathrm{L}^{-1}$, 结合 $(8)$ 式可得 $k=0.623 \mathrm{~L} \cdot \mathrm{mol}^{-1} \cdot \mathrm{s}^{-1}$, $k^{\prime}=7.85 \mathrm{~ms}^{-1}$, 由此可见碱性条件 $\left(0.05 \mathrm{~mol} \cdot \mathrm{L}^{-1} \mathrm{NaOH}\right.$ 溶液, $\left.\mathrm{pH}=12.7\right)$ 下, 酚酞在醌式(A)与甲 醇式(B)之间互相转化最终达到平衡状态。平衡浓度为: 醌式 $x_{\mathrm{A} \infty}=20.14 \mu \mathrm{mol} \cdot \mathrm{L}^{-1}$, 甲醇式 $x_{\mathrm{B} \infty}=$ $79.86 \mu \mathrm{mol} \cdot \mathrm{L}^{-1}$ 。

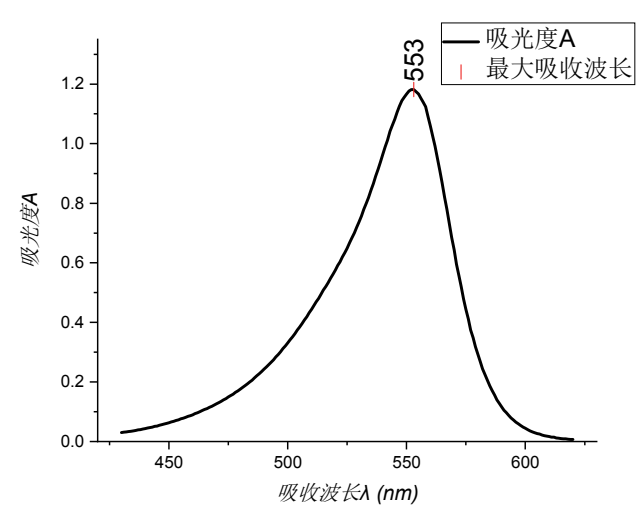

图2 $0.05 \mathrm{~mol} \cdot \mathrm{L}^{-1} \mathrm{NaOH}$ 溶液中酚酞 $A-\lambda$ 图

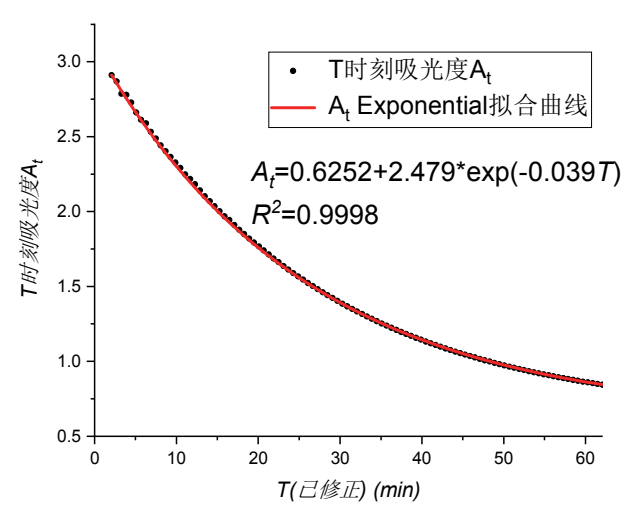

图3 $0.05 \mathrm{~mol} \cdot \mathrm{L}^{-1} \mathrm{NaOH}$ 溶液中酚酞 $A_{t}-T$ 图 


\section{4 酚酞浓度及温度对实验的影响}

\section{1 改变酚酞浓度获取各动力学曲线}

固定 $\mathrm{NaOH}$ 溶液为 $0.05 \mathrm{~mol} \cdot \mathrm{L}^{-1}$, 改变酚酞的加入量(见表 1 ), 重复步骤 3.3 , 将获得的数据用Origin 软件绘图, 并进行Exponential拟合(图4)。由图4可知, 酚酞浓度 $\leq 0.1 \mathrm{mmol} \cdot \mathrm{L}^{-1}$ 时, 数据曲线平滑性 好, 且点与回归曲线拟合度高, 与Nicholson的推论 ${ }^{[7]}$ 相符。酚酞浓度 $>0.1 \mathrm{mmol} \cdot \mathrm{L}^{-1}$ 时, 随着浓度增 大, 曲线平滑性越差, 实验前段时间数据越容易呈不规律变化。酚酞浓度 $\geq 0.5 \mathrm{mmol} \cdot \mathrm{L}^{-1}$ 时, 吸光度 集中在一个区间内振荡。综合考虑取用试剂、配制待测溶液的操作时间及曲线匹配性等因素, 建议 实验采用 $0.1 \mathrm{mmol} \cdot \mathrm{L}^{-1}$ 方案, 此时数据曲线平滑性好, 且吸光度较高, 即使学生在实验过程中操作速 度较慢, 也可以及时采集到需要的数据以便进行数据分析。

\section{2 改变体系温度获取各动力学曲线}

采用3.3的体系浓度, 改变体系温度(见表2), 重复步骤3.3, 将获得的数据用Origin软件绘图, 并 进行Exponential拟合(图5)。由图 5 可知, $25-40{ }^{\circ} \mathrm{C}$ 范围内, 数据曲线平滑性好, 且点与回归曲线拟合

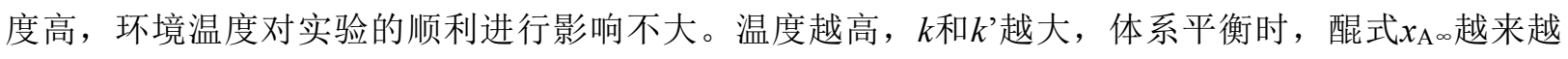
大, 甲醇式 $x_{\mathrm{B}} \infty$ 越来越小。

表1 图4中各曲线拟合情况说明

\begin{tabular}{cccc}
\hline 序号 & 酚酞乙醇溶液加入量 $/ \mathrm{mL}$ & 酚酞浓度 $/\left(\mathrm{mmol} \cdot \mathrm{L}^{-1}\right)$ & Origin中Exponential拟合曲线 \\
\hline$(1)$ & 0.05 & 0.05 & $A_{t}=0.3264+1.258 \exp (-0.040 T)$ \\
$(2)$ & 0.10 & 0.1 & $A_{t}=0.6252+2.479 \exp (-0.039 T)$ \\
$(3)$ & 0.15 & 0.15 & $A_{t}=0.7391+2.950 \exp (-0.037 T)$ \\
$(4)$ & 0.20 & 0.20 & $A_{t}=1.139+2.922 \exp (-0.38 T)$ \\
$(5)$ & 0.25 & 0.25 & 曲线平滑度不佳, 未作Exponential拟合 \\
$(6)$ & 0.50 & 0.5 & 曲线平滑度不佳, 未作Exponential拟合 \\
\hline
\end{tabular}

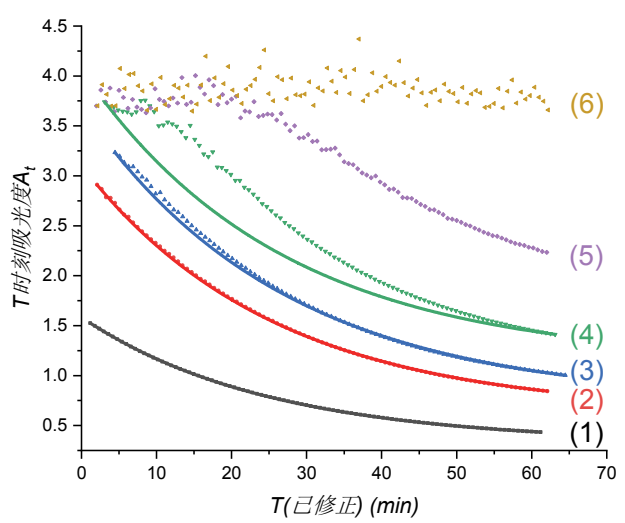

图4 不同酚酞浓度 $A_{t}-T$ 图 点为数据, 线为拟合曲线

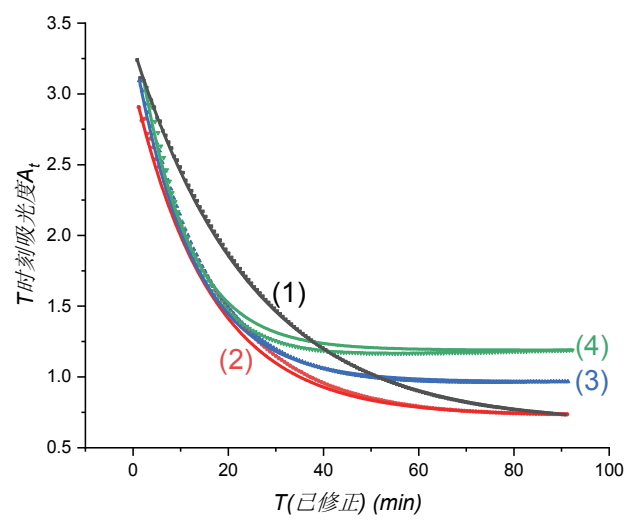

图5 不同温度 $A_{t}-T$ 图

点为数据, 线为拟合曲线

表2 图5中各曲线拟合情况说明

\begin{tabular}{cccccccc}
\hline 序号 & 温度 $/{ }^{\circ} \mathrm{C}$ & Origin中Exponential拟合曲线 & $R^{2}$ & $k /\left(\mathrm{L} \cdot \mathrm{mol}^{-1} \cdot \mathrm{s}^{-1}\right)$ & $k^{\prime} / \mathrm{ms}^{-1}$ & $x_{\mathrm{A} \infty} /\left(\mu \mathrm{mol} \cdot \mathrm{L}^{-1}\right)$ & $x_{\mathrm{B} \infty} /\left(\mu \mathrm{mol} \cdot \mathrm{L}^{-1}\right)$ \\
\hline$(1)$ & 25 & $A_{t}=0.6252+2.4791 \exp (-0.039 T)$ & 0.9998 & 0.623 & 7.85 & 20.14 & 79.86 \\
$(2)$ & 30 & $A_{t}=0.7130+2.400 \exp (-0.057 T)$ & 0.9997 & 0.879 & 13.1 & 22.90 & 77.10 \\
$(3)$ & 35 & $A_{t}=0.9470+2.443 \exp (-0.075 T)$ & 0.9993 & 1.08 & 21.0 & 27.94 & 72.06 \\
$(4)$ & 40 & $A_{t}=1.163+2.618 \exp (-0.105 T)$ & 0.9972 & 1.45 & 32.3 & 30.76 & 69.24 \\
\hline
\end{tabular}




\section{5 结语}

综上可知, 分光光度法研究酚酞 $-\mathrm{NaOH}$ 体系可逆动力学反应过程, 其最佳反应体系是在 $50 \mathrm{~mL}$ $0.05 \mathrm{~mol} \cdot \mathrm{L}^{-1} \mathrm{NaOH}$ 溶液中加入 $0.1 \mathrm{~mL} 0.05 \mathrm{~mol} \cdot \mathrm{L}^{-1}$ 酚酞乙醇溶液, 此体系使用的试剂毒性低、用量少, 符合绿色化学实验理念。温度 $\left(25-40{ }^{\circ} \mathrm{C}\right.$ 条件下) 只影响反应速率, 对反应趋势的影响不大, 该实验可 在大部分环境条件开展, 体系测量波长为 $553 \mathrm{~nm}$, 只用分光光度计即可满足实验条件。由于实验开 展条件灵活度高, 比如可以设计将学生适当分组, 采用多台分光光度计进行平行实验, 或设计不同 的实验温度进行采样等等, 适合不同的实验室条件、开课学生数及实验课时要求开展实验教学。

\section{参 考 文 献}

[1] 陈六平, 戴宗. 现代化学实验与技术. 第2版. 北京: 科学出版社, 2015: 161-187.

[2] 金丽萍, 鸟时清. 物理化学实验. 上海: 华东理工大学出版社, 2016: 41-52.

[3] 蔡维平. 基础化学实验(一). 北京: 科学出版社, 2004: 158-162.

[4] 丁益民, 张小平. 物理化学实验. 北京: 化学工业出版社, 2018: 55-68.

[5] 王红, 刘秋平, 王海滨, 杨振平, 强根荣. 实验室研究与探索, 2019, 38 (7), 139.

[6] 韩东梅, 宋树芹, Gewirtz, O.; Martin, A., 黄希哲. 基础化学实验. 广州: 中山大学出版社, 2017: 73-78.

[7] Nicholson, L. J. Chem. Ed. 1989, 66 (9), 725.

[8] 朱泽琛. 化学工程与装备, 2010, No. 12, 5. 